Response and adaptation. In a typical assay, a model system was subject to a step-like change in attractant concentration. A system in steady-state, characterized by the system activity $A^{\text {st }}$, was perturbed by an addition or removal of attractant. As a result, the system activity changed abruptly and then relaxed, with the characteristic adaptation time, $\tau$, to a new steady-state value $A^{\text {st }} . p$. Here $p$ measures the precision of adaptation; perfect adaptation corresponds to $p=1$ (see inset in Fig. 3a).

Robustness of adaptation. The sensitivity of adaptation precision and adaptation time to variations in the biochemical constants defining a model system was investigated. An ensemble of altered systems was obtained from the reference system by random modifications of its reaction rate constants and enzymatic concentrations, $k_{n}^{0}$. Each alternation of the reference system was characterized by the total parameter variation, $k$, which is defined as: $\log (k)=\Sigma_{n=1}^{L}\left|\log \left(k_{n} / k_{n}^{0}\right)\right|$, where $k_{n}$ are the biochemical parameters of the altered system. The altered system was subject to a step-like addition of saturating concentrations of attractant $(1 \mathrm{mM})$, and both the precision of adaptation, $p$, and the adaptation time, $\tau$, were measured. The assay was repeated for various reference model systems, with different values of biochemical parameters and of $\alpha_{m}$, and different variants of the model. The robustness of adaptation (Fig. 3) is independent of these choices.

Chemotactic drift velocity. The behaviour of a model system in the presence of a linear gradient of attractant, $\nabla l$, was simulated. The movement of the system was assumed to be composed of a series of smooth runs at a constant velocity of $20 \mu \mathrm{m} \mathrm{s}^{-1}$, interrupted by tumbling events. The tumbling frequency was taken to be a sigmoidal function of the system activity (Hill coefficient, $q=2$. Different values of $q$ lead to the same qualitative picture; the sensitivity increases with $q$ ). The trajectories were also subject to a rotation diffusion, with $D=0.125 \mathrm{rad}^{2} \mathrm{~s}^{-1}$ (ref. 9). Attractant concentration was increasing along the $x$ direction, (with $l=1 \mu \mathrm{M}$ at $x=0$ ). The chemotactic drift velocity was estimated by measuring the average $x$ position of a hundred identical simulated systems as a function of time.

Received 31 December 1996; accepted 17 April 1997.

1. Bray, D. Protein molecules as computational elements in living cells. Nature 376, 307-312 (1995).

2. Stock, J. B. \& Surette, M. in E. coli and S. typhimurium: Cellular and Molecular Biology (ed. Neidhardt, F. C.) 1103-1129 (American Soceity of Microbiology, Washington DC, 1996).

3. Parkinson, J. S. Signal transduction schemes of bacteria. Cell 73, 857-871 (1993).

4. Hazelbauer, G. L., Berg, H. C. \& Matsumura, P. M. Bacterial motility and signal transduction. Cell 73, 15-22 (1993).

5. Bourret, R. B., Borkovich, K. A. \& Simon, M. I. Signal transduction pathways involving protein phosphorylation in prokaryotes. Annu. Rev. Biochem. 60, 401-441 (1991).

6. Adler, J. Chemotaxis in bacteria. Annu. Rev. Biochem. 44, 341-356 (1975).

7. Bray, D., Bourret, R. B. \& Simon, M. I. Computer simulation of the phosphorylation cascade controlling bacterial chemotaxis. Mol. Biol. Cell 4, 469-482 (1993).

8. Adler, J. Chemotaxis in bacteria. Science 153, 708-716 (1996).

9. Berg, H. C. \& Brown, D. A. Chemotaxis in E. coli analysed by three-dimensional tracking. Nature 239, 500-504 (1972).

10. Macnab, R. M. \& Koshland, D. E. The gradient-sensing mechanism in bacterial chemotaxis. Proc. Nat Acad. Sci. USA 69, 2509-2512 (1972).

11. Block, S. M., Segall, J. E. \& Berg, H. C. Impulse responses in bacterial chemotaxis. Cell 31, 215-226 (1982).

12. Koshland, D. E. A response regulator model in a simple sensory system. Science 196, 1055 (1977).

13. Berg, H. C. \& Tedesco, P. M. Transient response to chemotactic stimuli in E. coli. Proc. Natl Acad. Sci. USA 72, 3235-3239 (1975)

14. Springer, M. S., Goy, M. F. \& Adler, J. Protein methylation in behavioural control mechanism and in signal transduction. Nature 280, 279-284 (1979).

15. Koshland, D. E., Goldbeter, A. \& Stock, J. B. Amplification and adaptation in regulatory and sensory systems. Science 217, 220-225 (1982).

16. Khan, S., Spudich, J. L., McCray, J. A. \& Tentham, D. R. Chemotactic signal integration in bacteria. Proc. Natl Acad. Sci. USA 92, 9757-9761 (1995).

17. Segel, L. A., Goldbeter, A., Devreotes, P. N. \& Knox, B. E. A mechanism for exact sensory adaptation based on receptor modification. J. Theor. Biol. 120,151-179 (1986).

18. Hauri, D. C. \& Ross, J. A model of excitation and adaptation in bacterial chemotaxis. Biophys. J. 68 , 708-722 (1995)

19. Asakura, S. \& Honda, H. Two-state model for bacterial chemoreceptor proteins. J. Mol. Biol. 176, 349367 (1984).

20. Spudich, J. L. \& Koshland, D. E. Non-genetic individuality: chance in the single cell. Nature 262, 467 471 (1976)

21. Kleene, S. J., Hobson, A. C. \& Adler, J. Attractants and repellents influence methylation and demethylation of methyl-accepting proteins in an extract of E. coli. Proc. Natl Acad. Sci. USA 76, 6309-6313 (1979).

Acknowledgements. We thank J. Stock, M. Surette, A. C. Maggs, U. Alon, L. Hartwell, M. Kirschner A. Levine, A. Libchaber, A. Murray and T. Surrey for discussion; A. C. Maggs for help with numerical issues; and J. Stock, M. Surette and H. Berg for introducing us to bacterial chemotaxis and pointing out many useful references. This work has been partially supported by grants from the NIH and the NSF. N.B. is a Rothschild Fellow and a Dicke Fellow at Princeton University.

Correspondence and requests for materials should be addressed to S.L. (e-mail: leibler@princeton.edu).

\section{A family of cytokine-inducible inhibitors of signalling}

\author{
Robyn Starr ${ }^{\star}$ Tracy A. Willson, Elizabeth M. Viney, \\ Leecia J. L. Murray ${ }^{\star}$, John R. Rayner†, \\ Brendan J. Jenkins $\dagger$, Thomas J. Gonda †, \\ Warren S. Alexander*, Donald Metcalf*, \\ Nicos A. Nicola $*$ \& Douglas J. Hilton*
}

* The Walter and Eliza Hall Institute for Medical Research and The Cooperative Research Center for Cellular Growth Factors, Parkville, Victoria, Australia 3052 $\dagger$ The Hanson Centre for Cancer Research, IMVS, Adelaide, Southern Australia, Australia 5000

Cytokines are secreted proteins that regulate important cellular responses such as proliferation and differentiation ${ }^{1}$. Key events in cytokine signal transduction are well defined: cytokines induce receptor aggregation, leading to activation of members of the JAK family of cytoplasmic tyrosine kinases. In turn, members of the STAT family of transcription factors are phosphorylated, dimerize and increase the transcription of genes with STAT recognition sites in their promoters ${ }^{1-4}$. Less is known of how cytokine signal transduction is switched off. We have cloned a complementary DNA encoding a protein SOCS-1, containing an SH2-domain, by its ability to inhibit the macrophage differentiation of M1 cells in response to interleukin-6. Expression of SOCS-1 inhibited both interleukin-6-induced receptor phosphorylation and STAT activation. We have also cloned two relatives of SOCS-1, named SOCS-2 and SOCS-3, which together with the previously described CIS (ref. 5) form a new family of proteins. Transcription of all four SOCS genes is increased rapidly in response to interleukin-6, in vitro and in vivo, suggesting they may act in a classic negative feedback loop to regulate cytokine signal transduction.

To identify cDNAs encoding proteins capable of suppressing cytokine signal transduction, we used an expression cloning approach. The strategy used the murine monocytic leukaemic M1 cell line that differentiates into mature macrophages and ceases proliferation in response to various cytokines, including interleukin-6 (IL-6), and in response to the steroid, dexamethasone ${ }^{6,7}$. Parental M1 cells were infected with the RUFneo retrovirus, into which a library of cDNAs from the factor-dependent haemopoietic cell line FDC-P1 had been inserted ${ }^{8}$. Retrovirally infected M1 cells that were unresponsive to IL-6 were selected in semi-solid agar culture by their ability to generate compact colonies in the presence of IL-6 and geneticin. One stable IL-6-unresponsive clone, 4A2, was obtained after examining $10^{4}$ infected cells (Fig. 1). A 1.4 kilobase pair (kbp) cDNA insert, which we have named suppressor of cytokine signalling-1, or SOCS-1, was recovered by polymerase chain reaction (PCR) from the retrovirus that had integrated into genomic DNA of $4 \mathrm{~A} 2$ cells. The SOCS-1 PCR product was used to
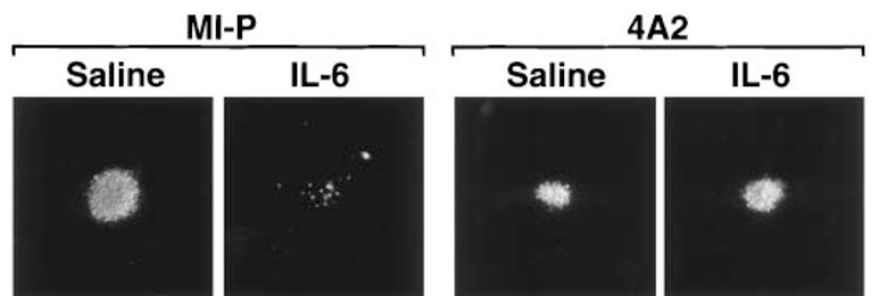

Figure 1 Phenotype of IL-6 unresponsive M1 cell clone, 4A2. Colonies of parental M1 cells (left panel) and clone 4A2 (right panel) cultured in semi-solid agar for 7 days in saline or $100 \mathrm{ng} \mathrm{ml}^{-1} \mathrm{IL}-6$. 
clone independent cDNAs from a mouse thymus cDNA library and these were identical in sequence to the retroviral cDNA insert, suggesting that constitutive expression of the wild-type SOCS-1 gene, rather than expression of a mutated cDNA, was sufficient to confer the IL-6-unresponsive phenotype.

A search of the nucleotide sequence databases revealed that the SOCS-1 gene was encoded on a single exon present in mouse and rat genomic DNA clones containing the protamine gene cluster found in the mouse on chromosome 16 (ref. 9). The SOCS-1 sequence was not homologous to any of the protamine genes, but corresponded to a previously unidentified open reading frame located at the $3^{\prime}$ end of these clones. Using sequence information provided by human expressed sequence tags (ESTs) several independent cDNAs encoding human SOCS-1 were also cloned. The mouse and rat SOCS-1 genes encode proteins of 212 amino acids, whereas the human gene encodes a protein of 211 amino acids. Mouse, rat and human SOCS-1 proteins share 95-99\% amino-acid identity (Fig. 2).

A search of translated nucleic acid databases with the predicted amino-acid sequence of SOCS-1 showed that it was distantly related to a recently cloned cytokine-inducible immediate early gene product, CIS (ref. 5) and to two further classes of ESTs. Using the sequences provided by the ESTs, we have cloned full-length cDNAs encoding related proteins we term SOCS-2 and SOCS-3. Although the amino-terminal regions of these proteins share little sequence similarity, all four contain a central $\mathrm{SH} 2$ domain and a conserved carboxy-terminal region that we have designated the SOCS box.
Mouse SOCS-1, SOCS-2, SOCS-3 and CIS appear quite distantly related. SOCS-2 and CIS exhibit approximately 38\% amino-acid identity, and the remaining members of the family share approximately $25 \%$ amino-acid identity (Fig. 2). Further evidence of the divergence of these genes is their distinct genomic organization. The coding regions of the genes for SOCS-1 and SOCS-3 appear to contain no introns, whereas the coding regions of the genes for SOCS-2 and CIS contain one and two introns, respectively (data not shown).

Although the function of the SOCS box is unknown, the core conserved sequence $(\mathrm{K} / \mathrm{R})(\mathrm{D} / \mathrm{E})(\mathrm{Y} / \mathrm{F})$ is similar to the tyrosine phosphorylation site in the JAK family (KEYY in Jak2) that regulates kinase activity ${ }^{10}$. Because this site is thought to act as a pseudosubstrate sequence in the unphosphorylated state, it is possible that SOCS proteins may inhibit JAK kinase activity through the SOCS box. This notion is supported by the observation that SOCS-1 interacts with the catalytic region of JAK kinases (see accompanying paper $\left.^{11}\right)$.

To establish that the phenotype of the $4 \mathrm{~A} 2$ cell line was directly related to expression of SOCS-1, and not to unrelated genetic changes that may have occurred in this cell clone, a cDNA encoding an epitope-tagged version of SOCS-1 was cloned into an expression vector under the control of the EF1 $\alpha$ promoter. The SOCS-1 expression vector was cotransfected with a plasmid conferring resistance to puromycin into parental M1 cells and, in order to assess the effect of SOCS-1 expression on signalling by a broader

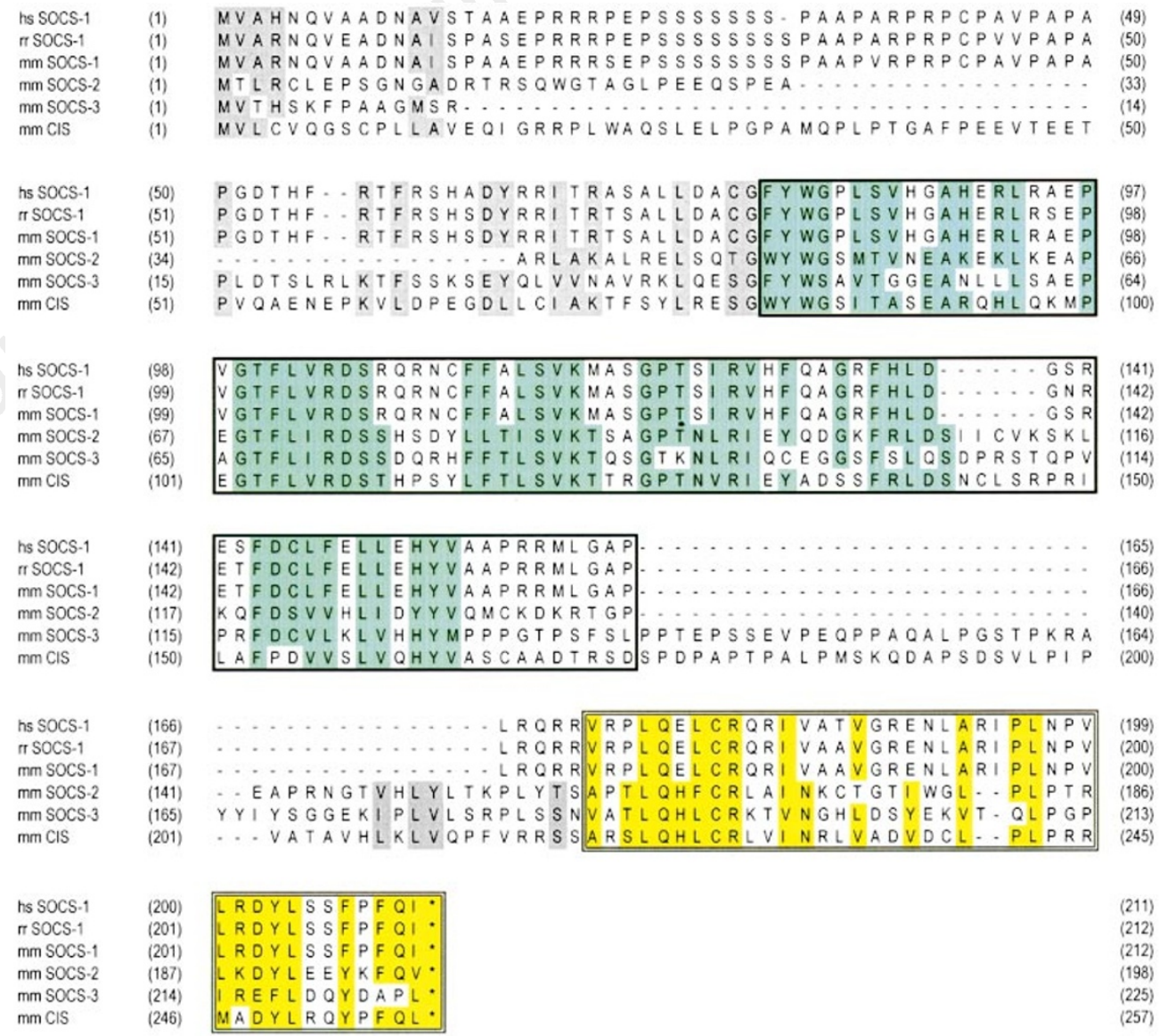

Figure 2 Comparison of the amino-acid sequences of SOCS-1, SOCS-2, SOCS-3 and $\mathrm{CIS}$. Alignment of the predicted amino-acid sequence of the mouse ( $\mathrm{mm}$ ), human (hs) and rat (rr) SOCS-1, SOCS-2, SOCS-3 and CIS. Those residues shaded are conserved in three of the four mouse SOCS family members. The $\mathrm{SH} 2$ domain is boxed in solid lines, whereas the SOCS box is bounded by double lines. The nucleotide sequence of each of these genes has been deposited in GenBank under the accession numbers mmSOCS-1 = U88325; hsSOCS-1 = U88326; mmSOCS-2 = U88327; mmSOCS-3 = U88328. 
range of cytokines, into M1 cells expressing the receptor for thrombopoietin, c-mpl (M1.mpl). Multiple independent clones of M1 cells expressing SOCS-1, as detected by western blot, displayed a cytokine-unresponsive phenotype that was indistinguishable from 4A2 (data not shown). Further, if transfectants were not maintained in puromycin, expression of SOCS-1 was lost over time and cells regained their cytokine responsiveness. In the absence of cytokine, colonies derived from 4A2 and other SOCS-1-expressing clones characteristically grew to a smaller size than colonies formed by control M1 cells (Fig. 1).

The effect of constitutive SOCS-1 expression on the response of $\mathrm{M} 1$ cells to a range of cytokines was further investigated using the $4 \mathrm{~A} 2$ cell line and a clone of M1.mpl cells expressing SOCS-1 (M1.mpl.SOCS-1). Unlike parental M1 cells and M1.mpl cells, the two cell lines expressing SOCS-1 continued to proliferate and failed to form differentiated colonies in response to either IL-6, leukaemia inhibitory factor (LIF), oncostatin M (OSM), interferon- $\gamma$ (IFN- $\gamma$ ) or, in the case of the M1.mpl.SOCS-1 cell line, thrombopoietin (Fig. 3). However, both cell lines were able to respond to dexamethasone, suggesting that SOCS-1 specifically affected cytokine signal transduction rather than the cell's intrinsic ability to differentiate. Consistent with these data, 4A2 and M1.mpl.SOCS-1 cells showed no evidence of morphological differentiation in response to IL- 6 or other cytokines, whereas parental M1 cells and M1.mpl cells became large and vacuolated with a macrophage morphology in response to IL-6 (data not shown).

Because CIS was cloned as a cytokine-inducible immediate early gene, we examined whether SOCS-1, SOCS-2 and SOCS-3 were similarly regulated. The basal pattern of expression of the four SOCS genes was examined by northern blot analysis of messenger RNA from a variety of tissues from male and female C57BL/6 mice (Fig. 4a). Constitutive expression of SOCS-1 was observed in the thymus and to a lesser extent in the spleen and the lung. SOCS-2 expression was restricted primarily to the testis and in some animals the liver and lung; for SOCS-3 a low level of expression was observed

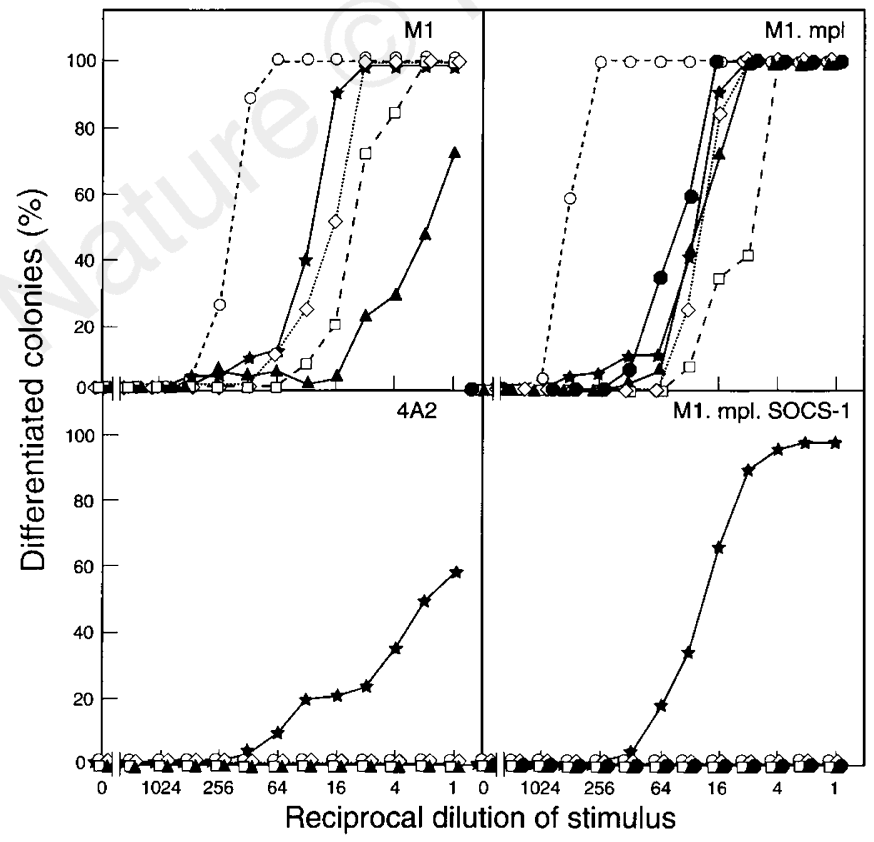

Figure 3 Expression of SOCS-1 suppresses IL-6-induced macrophage differentiation of M1 cells. Semi-solid agar cultures of parental M1 cells (M1 and M1.mpl) and $M 1$ cells expressing SOCS-1 (4A2 and M1.mpl.SOCS-1), showing the percentage of colonies that differentiated in response to a titration of $1 \mu \mathrm{g} \mathrm{ml}^{-1} \mathrm{IL}$ 6 (empty circles), $100 \mathrm{ng} \mathrm{ml}^{-1}$ LIF (empty triangles), $1 \mu \mathrm{g} \mathrm{ml}^{-1}$ OSM (squares), $100 \mathrm{ng} \mathrm{ml}^{-1} \mathrm{IFN}-\gamma$ (filled triangles), $500 \mathrm{ng} \mathrm{ml}^{-1}$ thrombopoietin (TPO) (filled circles) or $3 \times 10^{-6} \mathrm{M}$ dexamethasone (stars). in the lung, spleen and thymus, whereas CIS expression was more widespread, including the testis, heart, lung, kidney and, in some animals, the liver.

We sought to determine whether expression of the four SOCS genes was regulated by IL-6. Northern blots of mRNA prepared from the livers of untreated and IL-6-injected mice, or from unstimulated and IL-6-stimulated M1 cells, were hybridized with labelled fragments of SOCS-1, SOCS-2, SOCS-3 and CIS cDNAs (Fig. 4b). Expression of all four SOCS genes was increased in the liver following IL-6 injection; however, the kinetics of induction appeared to differ. Expression of SOCS-1 and SOCS-3 was transient in the liver, with mRNA detectable 20 min after IL-6 injection and declining to basal levels within $4 \mathrm{~h}$ for SOCS-1 and $8 \mathrm{~h}$ for SOCS-3. Induction of SOCS-2 and CIS mRNA in the liver followed similar initial kinetics to that of SOCS-1, but was maintained at an elevated level for at least $24 \mathrm{~h}$. A similar induction of SOCS gene mRNA was observed in other organs, notably the lung and the spleen (data not shown). In contrast, in M1 cells although SOCS-1 and CIS mRNA were induced by IL-6, no induction of either SOCS-2 or SOCS-3 expression was detected. This result highlights cell-type-specific differences in the expression of the genes of SOCS family members in response to the same cytokine.

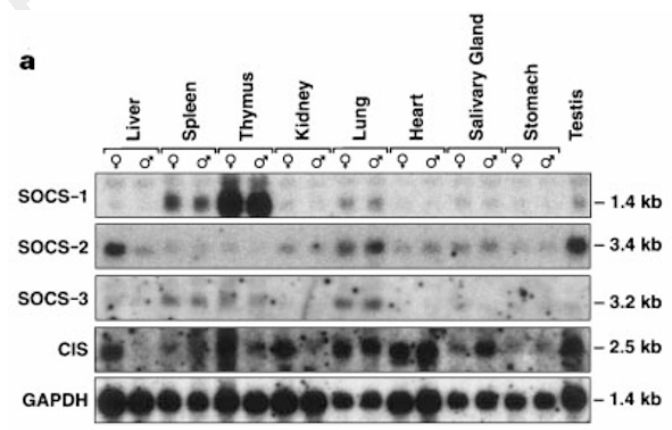

b
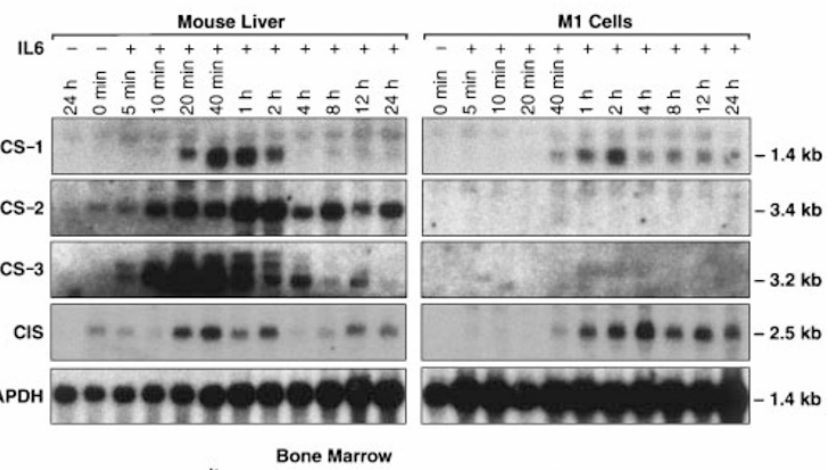

c

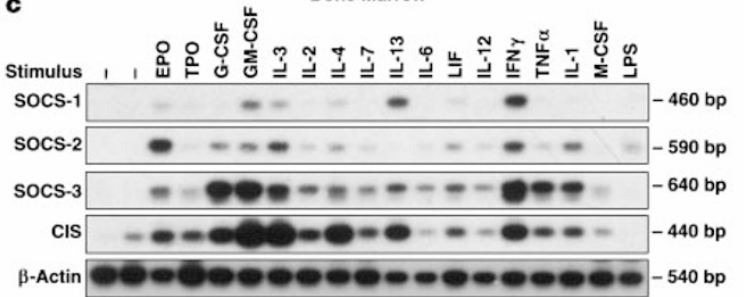

Figure 4 Expression of mRNA for SOCS family members in vitro and in vivo. a, Northern analysis of mRNA from a range of mouse organs showing constitutive expression of SOCS family members in a limited number of tissues. $\mathbf{b}$, Northern analysis of mRNA from liver and M1 cells showing induction of expression of SOCS family members following exposure to IL-6. c, Reverse transcriptase PCR analysis of mRNA from bone marrow showing induction of expression of SOCS family members by a range of cytokines. 
In order to examine the spectrum of cytokines that was capable of inducing transcription of the various members of the SOCS gene family, bone marrow cells were stimulated for an hour with a range of cytokines, after which mRNA was extracted and cDNA was synthesized. PCR was then used to assess the expression of SOCS1, SOCS-2, SOCS-3 and CIS (Fig. 4c). In the absence of stimulation, little or no expression of any of the SOCS genes was detectable in bone marrow by PCR. Stimulation of bone marrow cells with a broad array of cytokines appeared capable of upregulating mRNA for one or more members of the SOCS family. IFN- $\gamma$, for example, induced expression of all four SOCS genes, whereas erythropoietin, granulocyte colony-stimulating factor, granulocyte-macrophage colony-stimulating factor and interleukin-3 induced expression of SOCS-2, SOCS-3 and CIS. Interestingly, tumour necrosis factor- $\alpha$, macrophage colony-stimulating factor and interleukin-1, which act through receptors that do not fall into the type I cytokine receptor class also appeared capable of inducing expression of SOCS-3 and CIS, suggesting that the SOCS proteins may play a broader role in regulating signal transduction.

As constitutive expression of SOCS-1 inhibited the response of M1 cells to a range of cytokines, we examined whether phosphorylation of the cell-surface receptor component gp130 and the a

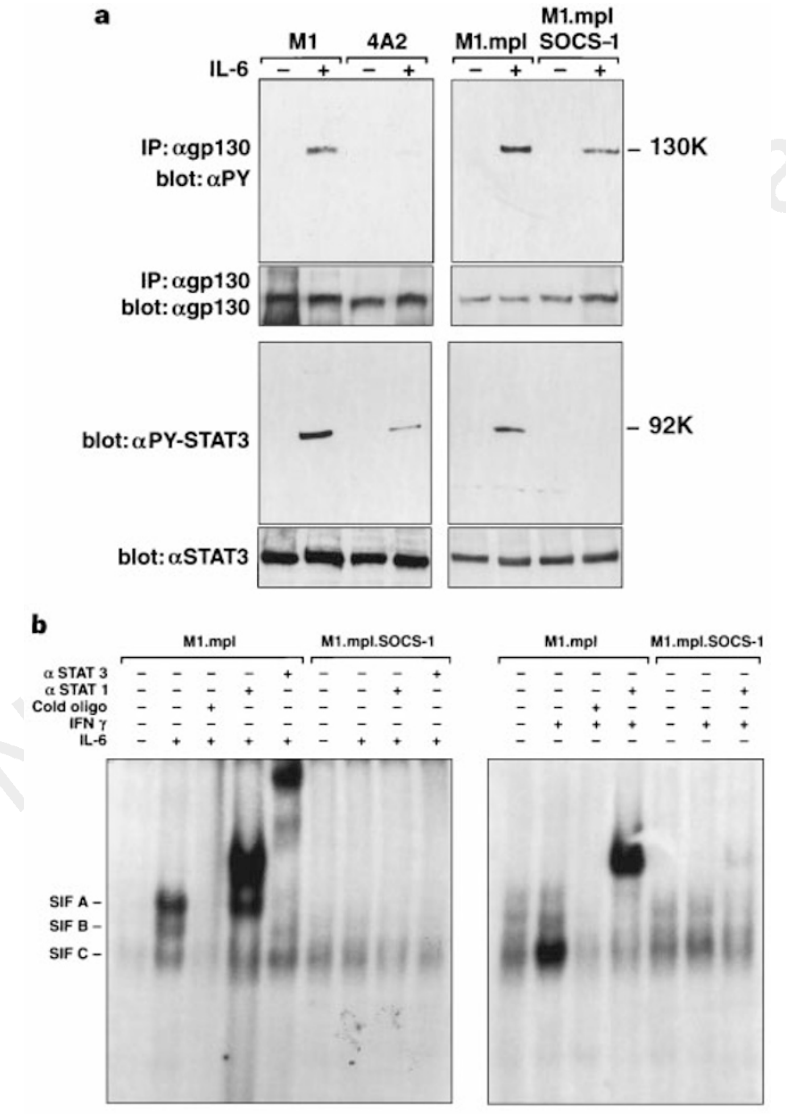

Figure 5 SOCS-1 suppresses the phosphorylation and activation of gp130 and Stat-3. a, Western blots of extracts from parental M1 cells (M1 and M1.mpl) and M1 cells expressing SOCS-1 (4A2 and M1.mpl.SOCS-1) stimulated with (+) or without ( - ) $100 \mathrm{ng} \mathrm{ml}^{-1}$ IL-6. Top: extracts immunoprecipitated with anti-gp130 $(\alpha \mathrm{gp} 130)$ and immunoblotted with anti-phosphotyrosine ( $\alpha \mathrm{PY})$. The blot was reprobed with $\alpha$ gp130 to demonstrate equal loading of protein. Bottom: cell lysates $(10 \mu \mathrm{g})$ immunoblotted either with an antibody specific for tyrosine phosphorylated-STAT3 ( $\alpha$ PY-Stat3), or for Stat3 ( $\alpha$ Stat3) to demonstrate equal loading of protein. The molecular masses of the bands are shown on the right. $\mathbf{b}$, EMSA of M1.mpl and M1.mpl.SOCS-1 cells stimulated with (+) and without ( - ) $100 \mathrm{ng} \mathrm{ml}^{-1} \mathrm{IL}-6$ or $100 \mathrm{ng} \mathrm{ml}^{-1} \mathrm{IFN}-\gamma$. The DNA-binding complexes SIFA, B, and C are indicated at the left. transcription factor Stat3, which are thought to play a central role in IL-6 signal transduction ${ }^{1-4}$, were affected. We compared these events in the parental M1 and M1.mpl cell lines and their SOCS-1expressing counterparts. As expected, gp130 was phosphorylated rapidly in response to IL-6 in both parental lines, however, this was reduced in the cell lines expressing SOCS-1 (Fig. 5a). Likewise, Stat3 phosphorylation was also reduced in response to IL- 6 in those cell lines expressing SOCS-1 (Fig. 5a). Consistent with a reduction in Stat3 phosphorylation, activation of specific STAT/DNA-binding complexes, as determined by electrophoretic mobility shift assay, was also reduced. Notably, there was a failure to form SIF-A (containing Stat3) and SIF-B (Stat1/Stat3 heterodimer), the major STAT complexes induced in M1 cells stimulated with IL-6 (Fig. 5b). Similarly, constitutive expression of SOCS-1 also inhibited IFN- $\gamma$ stimulated formation of SIF-C (Stat1 homodimer; Fig. 5b).

The effects of SOCS-1 appeared to be specific for the JAK/STAT pathway because no detectable diminution of the overall level of tyrosine-phosphorylated proteins was observed either in the absence or presence of IL-6 in M1 cells expressing SOCS-1 (data not shown). Similarly in M1 cells, which express flt3/flk-2 (ref. 12), flt3/flk-2 ligand-induced tyrosine phosphorylation of its receptor and the cytoplasmic protein Shc were unaffected by expression of SOCS-1 (data not shown). Taken together, these experiments are consistent with the proposal that SOCS-1 specifically inhibits signal transduction upstream of receptor and STAT phosphorylation. SOCS-1 may therefore act as a specific inhibitor of JAK kinases, and because these enzymes have been implicated in the transmission of both differentiative and proliferative signals ${ }^{13}$, it is possible that SOCS-1 may also inhibit cytokine-induced cell proliferation.

The ability of SOCS-1 to inhibit signal transduction and ultimately the biological response to cytokines suggests that, like the SH2-containing phosphatase SHP-1 (refs 14, 15), the SOCS proteins play a central role in controlling the intensity and/or duration of a cell's response to a diverse range of extracellular stimuli by suppressing the signal transduction process. The evidence provided here and in accompanying papers suggests that the SOCS family acts in a classic negative feedback loop for cytokine signal transduction. Like other genes such as OSM, expression of genes encoding the SOCS proteins is induced by cytokines through the activation of STATs $^{16,17}$. Once expressed, it is proposed that the SOCS proteins inhibit the activity of JAKs and so reduce the phosphorylation of receptors and STATs, thereby suppressing signal transduction and any ensuing biological response. Importantly, inhibition of STAT activation will, over time, lead to a reduction in SOCS gene expression, allowing cells to regain responsiveness to cytokines. Assessment of the physiological role of SOCS-1, SOCS-2, SOCS-3 and CIS will be clarified further by generating mice that lack one or more members of this family.

Note added in proof: Elsewhere in this issue, JAK inhibitors similar to SOCS are reported under the names JAB ${ }^{11}$ and SSI-1 (ref. 27).

\section{Methods}

Generation of retroviral library and infection of $\mathbf{M} \mathbf{1}$ cells. A cDNA expression library of $10^{4}$ clones was constructed from the factor-dependent haemopoietic cell line FDC-P1 and used to infect M1 cells, essentially as described $^{8}$. Infected IL-6 unresponsive M1 cells were selected in agar cultures containing $400 \mathrm{~g} \mathrm{ml}^{-1}$ geneticin and $100 \mathrm{ng} \mathrm{ml}^{-1} \mathrm{IL}-6$.

Database searches and cloning of cDNAs. cDNA inserts were recovered from integrated retroviruses by PCR, as described ${ }^{8}$. Independent cDNA clones encoding mouse SOCS-1 were isolated from a murine thymus CDNA library essentially as described ${ }^{18}$. The nucleotide and predicted amino-acid sequence of mouse SOCS-1 cDNA was compared to databases using the BLASTN and TFASTA algorithms ${ }^{19-21}$. Oligonucleotides were designed from the ESTs encoding human SOCS-1 and mouse SOCS-2 and SOCS-3 and used to probe commercially available mouse thymus and spleen cDNA libraries essentially as described previously ${ }^{18}$. Sequencing was performed using an ABI automatic sequencer according to the manufacturer's instructions. 
Northern analysis and reverse transcription. Purification of poly $(\mathrm{A})^{+}$ mRNA, and northern blot hybridization were performed essentially as described $^{22}$. To assess the induction of SOCS genes by IL-6, mice (C57BL/6) were injected intravenously with $5 \mu \mathrm{g}$ IL- 6 followed by collection of the liver at the indicated timepoints after injection. M1 cells were cultured in the presence of $20 \mathrm{ng} \mathrm{ml}^{-1}$ IL-6 and collected at the indicated times. For RT-PCR analysis, bone marrow cells were collected as described ${ }^{23}$ and stimulated for $1 \mathrm{~h}$ at $37^{\circ} \mathrm{C}$ with $100 \mathrm{ng} \mathrm{ml}^{-1}$ of a range of cytokines. RT-PCR was performed on total RNA as described ${ }^{23}$. PCR products were resolved on an agarose gel and Southern blots were hybridized as described ${ }^{23}$ with probes specific for each SOCS family member. Expression of $\beta$-actin was assessed to ensure uniformity of amplification.

Western blotting and electrophoretic mobility shift assays. M1 cells $\left(10^{7}\right)$ or their derivatives were stimulated for $4 \mathrm{~min}$ at $37^{\circ} \mathrm{C}$ with either saline or $100 \mathrm{ng} \mathrm{ml}^{-1} \mathrm{IL}-6$. Cells were lysed and 1 to $2 \mathrm{mg}$ protein was immunoprecipitated with $4 \mu \mathrm{g}$ anti-gp130 antibody (M20; Santa Cruz Biotechnology, Santa Cruz, CA) essentially as described ${ }^{24}$. Western blots were performed using antityrosine phosphorylated STAT3 or anti-STAT3 (New England Biolabs, Beverly, MA), or anti-gp130 (Santa Cruz Biotechnology) as described ${ }^{25}$. Electrophoretic mobility shift assays were performed using the $\mathrm{m} 67$ oligonucleotide probe, as described $^{26}$.

Received 19 December 1996; accepted 21 March 1997.

1. Nicola, N. A. Guidebook to Cytokines and Their Receptors (Oxford Univ. Press, Oxford, 1994).

2. Ihle, J. N. Cytokine receptor signalling. Nature 377, 591-594 (1995).

3. Ihle, J. N., Witthuhn, B. A., Quelle, F. W., Yamamoto, K. \& Silvennoinen, O. Signaling through the hematopoietic cytokine receptors. Ann. Rev. Immunol. 13, 369-398 (1995).

4. Darnell, J. Jr, Kerr, I. M. \& Stark, G. R. Jak-STAT pathways and transcriptional activation in response to IFNs and other extracellular signaling proteins. Science 264, 1415-1421 (1994).

5. Yoshimura, A. et al. A novel cytokine-inducible gene CIS encodes an $\mathrm{SH} 2$-containing protein that binds to tyrosine-phosphorylated interleukin 3 and erythropoietin receptors. EMBO J. 14, 2816-2826 (1985).

6. Metcalf, D., Hilton, D. J. \& Nicola, N. A. Clonal analysis of the actions of the murine leukemia inhibitory factor on leukemic and normal murine hemopoietic cells. Leukemia 2, 216-221 (1988).

. Lotem, J., Shabo, Y. \& Sachs, L. Clonal variation in susceptibility to differentiation by different protein. Leukemia 3, 804-807 (1989)

8. Rayner, J. R. \& Gonda, T. J. A simple and efficient procedure for generating stable expression libraries by cDNA cloning in a retroviral vector. Mol. Cell. Biol. 14, 880-887 (1994).

9. Schlueter, G. et al. Sequence analysis of the conserved Protamine gene cluster shows that it contains a fourth expressed gene. Mol. Reprod. Dev. 43, 1-6 (1996).

10. Feng, J. et al. Activation of JAK2 catalytic activity requires phosphorylation of Y1007 in the kinase activation loop. Mol. Cell. Biol. (in the press).

11. Endo, T. A. et al. A new protein containing an SH2 domain that inhibits JAK kinases. Nature 387, $921-$ 924 (1997)

12. Rasko, J. E., Metcalf, D., Rossner, M. T., Begley, C. G. \& Nicola, N. A. The flt3/flk-2 ligand: receptor distribution and action on murine haemopoietic cell survival and proliferation. Leukemia 9, 20582066 (1995).

13. Smith, A., Metcalf, D. \& Nicola, N. A. Cytoplasmic domains of the common $\beta$-chain of the GM-CSF/ IL-3/IL-5 receptors that are required for inducing differentiation or clonal suppression in myeloid leukaemic cell lines. EMBO J. 16, 451-464 (1997).

14. Ihle, J. N. et al. Protein tyrosine phosphorylation in the regulation of hematopoiesis by receptors of the cytokine-receptor superfamily. Blood Cells 20, 65-80 (1994).

15. Yi, T., Mui, A. L., Krystal, G. \& Ihle, J. N. Hematopoietic cell phosphatase associates with the interleukin-3 (IL-3) receptor beta chain and down-regulates IL-3-induced tyrosine phosphorylation and mitogenesis. Mol. Cell. Biol. 13, 7577-7586 (1993).

16. Mui, A. L., Wakao, H., Kinoshita, T., Kitamura, T. \& Miyajima, A. Suppression of interleukin-3induced gene expression by a C-terminal truncated Stat5: role of Stat5 in proliferation. EMBO J. 15, 2425-2423 (1996).

17. Quelle, F. W. et al. Erythropoietin induces activation of Stat 5 through association with specific tyrosines on the receptor that are not required for a mitogenic response. Mol. Cell. Biol. 16, 1622-1631 (1996).

18. Hilton, D. J. et al. Cloning of a murine IL-11 receptor alpha-chain; requirement for gp 130 for high affinity binding and signal transduction. EMBO J. 13, 4765-4775 (1994).

19. Pearson, W. R. \& Lipman, D. J. Improved tools for biological sequence comparison. Proc. Natl Acad. Sci. USA 85, 2444-2448 (1988).

20. Pearson, W. R. Rapid and sensitive sequence comparison with FASTP and FASTA. Methods Enzymol. 183, 63-98 (1990)

21. Altschul, S. F., Gish, W., Miller, W., Myers, E. W. \& Lipman, D. J. Basic local alignment search tool. J. Mol. Biol. 215, 403-410 (1990).

22. Alexander, W. S., Metcalf, D. \& Dunn, A. R. Point mutations within a dimer interface homology domain of c-Mpl induce constitutive receptor activity and tumorigenicity. EMBO J. 14, 5569-5578 (1995).

23. Metcalf, D., Willson, T. A., Hilton, D. J., DiRago, L. \& Mifsud, S. Production of hematopoietic regulatory factors in cultures of adult and fetal mouse organs: measurement by specific bioassays. Leukaemia 9, 1556-1564 (1995).

24. Hilton, D. J., Watowich, S., Katz, L. \& Lodish, H. F. Saturation mutagenesis of the WSXWS motif of the erythropoietin receptor. J. Biol. Chem. 271, 4699-4708 (1996).

25. Nicola, N. A., Viney, E., Hilton, D. J., Roberts, B. \& Willson, T. Cloning of two novel transmembrane ligands for eph-related linases (LERKs) that are related to LERK2. Growth Factors 13, 141-149 (1996).

26. Novak, U. et al. Colony-stimulating factor 1-induced STAT1 and STAT3 activation is accompanied by phosphorylation of Tyk2 in macrophages and Tyk2 and JAK1 in fibroblasts. Blood 86, 2948-2956 (1995).

27. Naka, T. et al. Structure and function of a new STAT-induced STAT inhibitor. Nature 387, 924-929 (1997).
Acknowledgements. We thank R. Simpson and R. Moritz for recombinant mouse IL-6, C. McFarlane for help with production of recombinant cytokines, S. Cory for the PPGKpuropA vector, P. Lock for the pPGKneo vector and U. Novak for her generous advice regarding electrophoretic mobility shift assays. B. Roberts, D. Cary, L. Di Rago, S. Mifsud and N. Sprigg are thanked for their excellent technical assistance. D.J.H. was supported by a Queen Elizabeth II Postdoctoral Fellowship from the Australian Research Council. T.J.G. is a senior research fellow of the NH\&MRC. This work was supported by the Anti-Cancer Council of Victoria, Melbourne, Australia, AMRAD Operations Pty Ltd, Melbourne, Australia The National Health and Medical Research Council, Canberra, Australia, The J.D. and L. Harris Trust, the National Institutes of Health, Bethesda, Maryland and the Australian Federal GovernHarris Trust, the National Institutes of Health, Bet
ment Cooperative Research Centres Programme.

Correspondence and requests for materials should be addressed to D.J.H. (e-mail: hilton@wehi.edu.au)

\section{A new protein containing an SH2 domain that inhibits JAK kinases}

\section{Takaho A. Endo ${ }^{\star} \dagger$, Masaaki Masuhara ${ }^{\star} \dagger$, Masahiro Yokouchi ${ }^{\ngtr} \ddagger$, Ritsu Suzuki $¥ \ddagger$, Hiroshi Sakamoto*, Kaoru Mitsui, Akira Matsumoto*, Shyu Tanimura*, Motoaki Ohtsubo*, Hiroyuki Misawa*, Tadaaki Miyazaki $\varsigma$, Nogueira Leonor $\$$, Tadatsugu Taniguchis, Takashi Fujitall, Yuzuru Kanakurag, Seturo Komiya: \& Akihiko Yoshimura*}

* Institute of Life Science, and $\ddagger$ Department of Orthopedic Surgery,

Kurume University, Aikawamachi 2432-3 Kurume 839, Japan

$\$$ Department of Immunology, Faculty of Medicine, University of Tokyo,

Bunkyo-ku, Tokyo 113, Japan

$\|$ Department of Tumor Cell Biology, The Tokyo Metropolitan Institute of Medical Science, Bunkyouku, Tokyo 113, Japan

I Department of Hematology and Oncology, Osaka University Medical School, Suita 565, Japan

$\dagger$ These authors contributed equally to this work.

The proliferation and differentiation of cells of many lineages are regulated by secreted proteins known as cytokines. Cytokines exert their biological effect through binding to cell-surface receptors that are associated with one or more members of the JAK family of cytoplasmic tyrosine kinases. Cytokine-induced receptor dimerization leads to the activation of JAKs, rapid tyrosinephosphorylation of the cytoplasmic domains, and subsequent recruitment of various signalling proteins, including members of the STAT family of transcription factors, to the receptor complex $^{1-5}$. Using the yeast two-hybrid system, we have now isolated a new $\mathrm{SH}$ 2-domain-containing protein, JAB, which is a JAK-binding protein that interacts with the Jak2 tyrosine-kinase JH1 domain ${ }^{6}$. JAB is structurally related to CIS, a cytokineinducible $\mathrm{SH} 2$ protein $^{7,8}$. Interaction of JAB with Jak1, Jak2 or Jak3 markedly reduces their tyrosine-kinase activity and suppresses the tyrosine-phosphorylation and activation of STATs. $\mathrm{JAB}$ and CIS appear to function as negative regulators in the JAK signalling pathway.

We screened several yeast two-hybrid complementary DNA libraries and found a single positive clone, JAB, in a human B-cell library that could interact with the Jak2 JH1 domain (Fig. 1a-c). A database search revealed that the mouse and rat JAB genes are located downstream of the protamine gene cluster'. The coding region of the gene for mouse $\mathrm{JAB}$ appears to contain no introns, and encodes a 212-amino-acid protein. The JAB protein contains a central SH2 domain (amino acids 79-170) which is most closely related (35\% identical) to that of CIS, a cytokine-inducible SH2containing protein (Fig. 1d) that we have cloned previously ${ }^{7}$.

We examined the specificity of the interaction of JAB with the Jak2 JH1 domain using the yeast two-hybrid assay. We found no evidence for interaction of the Jak2 JH1 domain with other SH2containing proteins such as Crk, the $\mathrm{p} 85$ subunit of phosphatidylinositol-3-OH kinase (PI(3)K), phospholipase C- $\gamma(\mathrm{PLC} \gamma)$ or a new 